\title{
How to make the Sun look less like the Sun and more like a star?
}

\author{
A. A. Vidotto ${ }^{1}$ \\ ${ }^{1}$ School of Physics, Trinity College Dublin, the University of Dublin, Dublin-2, Ireland \\ email: Aline.Vidotto@tcd.ie
}

\begin{abstract}
Synoptic maps of the vector magnetic field have routinely been made available from stellar observations and recently have started to be obtained for the solar photospheric field. Although solar magnetic maps show a multitude of details, stellar maps are limited to imaging large-scale fields only. In spite of their lower resolution, magnetic field imaging of solar-type stars allow us to put the Sun in a much more general context. However, direct comparison between stellar and solar magnetic maps are hampered by their dramatic differences in resolution. Here, I present the results of a method to filter out the small-scale component of vector fields, in such a way that comparison between solar and stellar (large-scale) magnetic field vector maps can be directly made. This approach extends the technique widely used to decompose the radial component of the solar magnetic field to the azimuthal and meridional components as well, and is entirely consistent with the description adopted in several stellar studies. This method can also be used to confront synoptic maps synthesised in numerical simulations of dynamo and magnetic flux transport studies to those derived from stellar observations.
\end{abstract}

Keywords. stars: magnetic fields

\section{Introduction}

Direct comparison between stellar and solar magnetic maps are hampered by their dramatic differences in resolution. Figure 1a illustrates the vector synoptic map of the Sun (Gosain et al. 2013), which can be directly compared to the vector synoptic map of a star (Figure 1b). The difference between both maps can be immediate recognised: the solar magnetic field has a "salt-and-pepper" structure, with high intensity magnetic fields localised in very small regions, while the stellar map shows a more smooth distribution of magnetic fields. This happens because the stellar map cannot reach the spectacular resolution of solar observations and are limited to only the large scale surface magnetic fields. More quantitatively, if we use spherical harmonics (see next section) to decompose the magnetic field, a solar magnetic field map can be decomposed out to quite high maximum spherical harmonics degree $l_{\max }>190$ (DeRosa et al. 2012), while stellar maps such as the one shown in Figure $1 \mathrm{~b}$, would typically have $l_{\max } \lesssim 10$ (i.e., $l_{\max }$ is used as proxy for spatial resolution).

The goal of this work is to devise a way to compare high-resolution magnetic maps of the Sun with low-resolution maps of stars. This method was published in Vidotto (2016). In this article, I present a summary of the method and how it can be applied to compare solar and stellar magnetic field maps.

\section{The method and a case study}

Stellar magnetic maps can be observationally derived using the Zeeman Doppler Imaging (ZDI) technique (Donati \& Brown 1997). ZDI can reconstruct the three components of the magnetic fields (radial $r$, meridional $\theta$ and azimuthal $\varphi$ ), but due to flux cancelation 


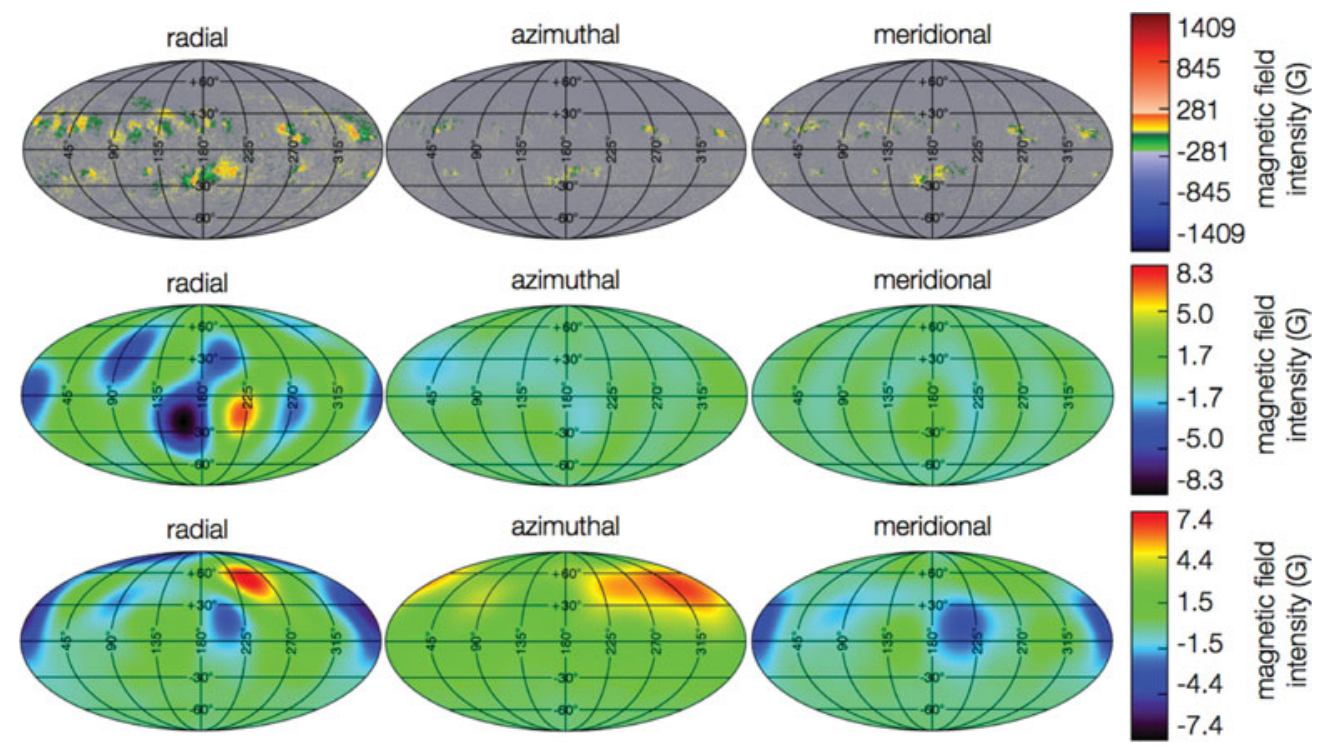

Figure 1. (a) Solar vector magnetic map (data from Gosain et al. 2013). (b) Vector magnetic map of a solar-type star where $l_{\max }=8$ (Fares et al. 2009). (c) The solar magnetic field as seen from a "stellar" perspective $l_{\max }=5$ (Vidotto 2016).

of small-scale fields within an element of resolution, ZDI is restricted to mapping only the large-scale magnetic field of the stellar surface (e.g. Lang et al. 2014). ZDI studies decompose the magnetic field using spherical harmonics as follows (Donati et al. 2006):

$$
\begin{gathered}
B_{r}(\theta, \varphi)=\sum_{\operatorname{lm}} \alpha_{\operatorname{lm}} P_{\operatorname{lm}} e^{i m \varphi}, \\
B_{\theta}(\theta, \varphi)=\sum_{\operatorname{lm}} \frac{\beta_{\operatorname{lm}}}{l+1} \frac{\mathrm{d} P_{\operatorname{lm}}}{\mathrm{d} \theta} e^{i m \varphi}+\gamma_{\operatorname{lm}} \frac{i m P_{\operatorname{lm}} e^{i m \varphi}}{(l+1) \sin \theta}, \\
B_{\varphi}(\theta, \varphi)=-\sum_{\operatorname{lm}} \beta_{\operatorname{lm}} \frac{i m P_{\operatorname{lm}} e^{i m \varphi}}{(l+1) \sin \theta}-\frac{\gamma_{\operatorname{lm}}}{l+1} \frac{\mathrm{d} P_{\operatorname{lm}}}{\mathrm{d} \theta} e^{i m \varphi},
\end{gathered}
$$

where $P_{\operatorname{lm}} \equiv P_{\operatorname{lm}}(\cos \theta)$ is the associated Legendre polynomial of degree $l$ and order $m$. $\alpha_{\mathrm{lm}}, \beta_{\mathrm{lm}}, \gamma_{\mathrm{lm}}$ are the coefficients that provide the best fit to the spectropolarimetric data, i.e., they are derived from ZDI studies. Therefore, once we have $\alpha_{l, m}, \beta_{l, m}$ and $\gamma_{l, m}$, we use equations (2.1) to (2.3) to derive the three components of the magnetic field.

The solar data, on the other hand, provide the three components of the magnetic field. If we want to compute the coefficients, following the formalism used in ZDI studies, we first need to invert equations (1) to (3) to obtain $\alpha_{l, m}, \beta_{l, m}$ and $\gamma_{l, m}$. The mathematical description to invert these equations is fully presented in Vidotto (2016). Given arrays of $\left\{B_{r}, B_{\theta}, B_{\varphi}\right\}$ spaced in $n_{\theta}$ latitudinal grid points and $n_{\varphi}$ longitudinal grid points, the real and imaginary parts of $\alpha_{l, m}, \beta_{l, m}$ and $\gamma_{l, m}$ are given by

$$
\Re\left(\alpha_{\mathrm{lm}}\right)=\sum_{i=1}^{n_{\varphi}} \sum_{j=1}^{n_{\theta}} B_{r}^{j i} P_{\operatorname{lm}}\left(\theta_{j}\right) \cos \left(m \varphi_{i}\right) \sin \theta_{j} \delta,
$$




$$
\begin{gathered}
\Im\left(\alpha_{\mathrm{lm}}\right)=-\sum_{i=1}^{n_{\varphi}} \sum_{j=1}^{n_{\theta}} B_{r}^{j i} P_{\mathrm{lm}}\left(\theta_{j}\right) \sin \left(m \varphi_{i}\right) \sin \theta_{j} \delta \\
\Re\left(\beta_{\mathrm{lm}}\right)=\sum_{i=1}^{n_{\varphi}} \sum_{j=1}^{n_{\theta}}\left\{B_{\theta}^{j i} \cos \left(m \varphi_{i}\right) \sin \theta_{j} \frac{\mathrm{d} P_{\mathrm{lm}}\left(\theta_{j}\right)}{\mathrm{d} \theta}+B_{\varphi}^{j i} m \sin \left(m \varphi_{i}\right) P_{\mathrm{lm}}\left(\theta_{j}\right)\right\} \frac{\delta}{l}, \\
\Im\left(\beta_{\mathrm{lm}}\right)=-\sum_{i=1}^{n_{\varphi}} \sum_{j=1}^{n_{\theta}}\left\{B_{\theta}^{j i} \sin \left(m \varphi_{i}\right) \sin \theta_{j} \frac{\mathrm{d} P_{\operatorname{lm}}\left(\theta_{j}\right)}{\mathrm{d} \theta}-B_{\varphi}^{j i} m \cos \left(m \varphi_{i}\right) P_{\operatorname{lm}}\left(\theta_{j}\right)\right\} \frac{\delta}{l}, \\
\Im\left(\gamma_{\mathrm{lm}}\right)=-\sum_{i=1}^{n_{\varphi}} \sum_{j=1}^{n_{\theta}}\left\{B_{\theta}^{j i} m \sin \left(m \varphi_{i}\right) P_{\operatorname{lm}}\left(\theta_{j}\right)-B_{\varphi}^{j i} \cos \left(m \varphi_{i}\right) \sin \theta_{j} \frac{\mathrm{d} P_{\operatorname{lm}}\left(\theta_{j}\right)}{\mathrm{d} \theta}\right\} \frac{\delta}{l}, \\
\left.n_{\varphi} \gamma_{\mathrm{lm}}\right)=-\sum_{i=1}^{n_{\theta}} \sum_{j=1}^{j i}\left\{B_{\theta}^{j i} m \cos \left(m \varphi_{i}\right) P_{\operatorname{lm}}\left(\theta_{j}\right)+B_{\varphi}^{j i} \sin \left(m \varphi_{i}\right) \sin \theta_{j} \frac{\mathrm{d} P_{\operatorname{lm}}\left(\theta_{j}\right)}{\mathrm{d} \theta}\right\} \frac{\delta}{l},
\end{gathered}
$$

where $\delta=\Delta \theta \Delta \varphi, \Delta \theta=\pi / n_{\theta}$ and $\Delta \varphi=2 \pi / n_{\varphi}$.

Therefore, from solar data (i.e., $B_{r}, B_{\theta}$ and $B_{\varphi}$ ), we can derive $\alpha_{l, m}, \beta_{l, m}$ and $\gamma_{l, m}$ for very large values of $l$-degrees using Equations (2.4) - (2.9). We then use these coefficients in Equations (1) to (3), but we limit the maximum value of $l$-degree in the sums to values similar to the ones reached in stellar ZDI studies. In other words, by restricting the sums to low- $l$ degrees, we can filter out the small-scale field, which is not assessable in ZDI stellar studies, and the solar "large-scale" map can then be directly compared to stellar maps. A filtered solar magnetic field map is shown in Figure 1c.

\section{Conclusions}

Here, I presented a method to filter out the small-scale component of vector fields, so that comparison between solar and stellar large-scale magnetic fields can be directly made in all three components. This approach is entirely consistent with the description adopted in several stellar studies.

The method can be used to confront synoptic maps synthesised in numerical simulations of dynamo and magnetic flux transport studies to those derived from stellar observations. A recent application of the method was presented in Lehmann et al. (2016).

\section{Acknowledgements}

The solar synoptic map in the top panel of Figure 1 was acquired by SOLIS instruments operated by NISP/NSO/AURA/NSF.

\section{References}

DeRosa, M. L., Brun, A. S., \& Hoeksema, J. T. 2012, ApJ, 757, 96

Donati, J., Howarth, I. D., Jardine, M. M., et al. 2006, MNRAS, 370, 629

Donati, J.-F. \& Brown, S. F. 1997, A\& A, 326, 1135

Fares, R., Donati, J., Moutou, C., et al. 2009, MNRAS, 398, 1383

Gosain, S., Pevtsov, A. A., Rudenko, G. V., \& Anfinogentov, S. A. 2013, ApJ, 772, 52

Lang, P., Jardine, M., Morin, J., et al. 2014, MNRAS, 439, 2122

Lehmann, L. T., Jardine, M. M., Vidotto, A. A., et al. 2016, MNRAS, in press. ArXiv: 1610.08314

Vidotto, A. A. 2016, MNRAS, 459, 1533 